\title{
TRAYECTORIAS DE LA DOMINACIÓN SOCIAL DE LOS ESPACIOS RURALES EN LA PROVINCIA DE LA PAMPA, ARGENTINA
}

\author{
Rodrigo Hernán Alves Rolo \\ Estudiante de la Maestría en Desarrollo Rural \\ Facultad de Agronomía.Universidad de Buenos Aires \\ Ciudad Autónoma de Buenos Aires, Argentina \\ Correo electrónico: rodrigoalvesrolo@gmail.com
}

Recibido: 25 de noviembre de 2011. Devuelto para revisión: 14 de diciembre de 2011.

Aceptado: 10 de enero de 2012

\begin{abstract}
RESUMEN
En el siguiente trabajo me propongo comprender la configuración espacial emergente como resultado del proceso de modernización capitalista de la estructura agraria en la Provincia de La Pampa. Este periodo se caracteriza por una fuerte tendencia a la concentración de la propiedad de la tierra, con disminución del número de productores por la desaparición de una gran cantidad de explotaciones ubicadas en los estratos de menores superficies. La profundización de las políticas neoliberales en la década del '90, etapa signada por la desregulación económica y la retracción del Estado, aceleró los cambios en la estructura socioeconómica agropecuaria, exacerbando el proceso de polarización social. Mientras la desregulación económica impactó espacialmente a través del incremento de los umbrales mínimos en la escala de producción para mejorar las condiciones de competitividad (1988-2002), la retracción del Estado facilitó el endeudamiento de muchos productores. El proceso de incorporación del territorio pampeano a los espacios del capital, así como su actualización, exige ser observado desde una perspectiva geohistórica que permita analizar la articulación trialéctica entre territorialización, desterritorialización y reterritorialización (TDR) de los sistemas de dominación social predominantes.
\end{abstract}

Palabras clave: Dinámica territorial, Estructura social agraria, Modernización tecnológica, Reorganización espacial del capital.

\section{RESUMO}

Neste artigo proponho a entender a configuração espacial emergente como resultado da modernização capitalista da estrutura agrária na província de La Pampa. Este período é caraterizado por uma forte tendência para a concentração da propriedade da terra, com uma diminuição no múmero de produtores por o desaparecimento de um grande número de explotações na superfície inferior. $\mathrm{O}$ aprofundamento das políticas neoliberais nos anos 90, uma etapa marcada pela desregulação econômica ea retirada do Estado, acelerou as mudanças na estruturasocioeconômica da agricultura, agravando o processo de polarização social. Enquanto a desregulação econômica impactou espacialmente, aumentando os limiares mínimos na escala de produção para melhorar a competitividade (1988-2002) a retração do Estado facilitou o endividamento de muitos agricultores. O processo de incorporação da pampa para os espaços do capital, e su atualização, exigem ser observados desde uma perspectiva geo-histórica para analisar a 
ligação trialéctica entre territorialização, desterritorialização e reterritorialização (TDR) dos sistemas prevalecentes da dominação social.

Palabras-chave: Dinâmica territorial, Estrutura social agrária, Modernização tecnológica, Reorganizaçâo espacial do capital.

\begin{abstract}
In this paper I propose to understand the emerging spatial configuration as a result of the capitalist modernization of the agrarian structure in the province of La Pampa. This period is characterized by a strong trend towards concentration of land ownership, with a decrease in the number of producers because of the disappearance of a large number of farms in the lower-surfaces. The deepening of neoliberal policies in the $90 \mathrm{~s}$, a stage marked by economic deregulation and the withdrawal of the State, accelerated changes in the socioeconomic structure of agriculture, exacerbating the process of social polarization. While economic deregulation impacted spatially by increasing the minimum thresholds in the production scale to improve the competitiveness (19882002), retraction of the State meant the indebtedness of many farmers. The process of incorporation of La Pampa's territory to the spaces of capital, as its updating, requires to be observed from a geo-historical perspective to analize the trialectical linking between territorialization, deterritorialization and reterritorialization (TDR) of the prevailing social domination systems.
\end{abstract}

Keywords: Territorial Dynamic, Agrarian Social Structure, Technological Modernization, Spatial Reorganization of Capital.

\title{
INTRODUCCIÓN
}

En el siguiente trabajo me propongo comprender la configuración espacial emergente como resultado del proceso de modernización capitalista de la estructura agraria en la Provincia de La Pampa. Para ello considero ineludible la descripción del proceso de apropiación del territorio por parte del estado nacional a partir de la llamada "Conquista del Desierto". La hipótesis que guía el trabajo apunta que, desde el conocimiento situado, la dinámica territorial actual puede pensarse como resultado de las continuas confrontaciones entre actores y grupos sociales con intereses contrapuestos respecto del proceso de actualización capitalista impuesto por el sistema de dominación social hegemónico.

La estructura del trabajo se compone de dos partes: la primera se subdivide en cuatro fases, a través de las que se describe el proceso de apropiación y ocupación del territorio pampeano. En la segunda parte se analizan distintas perspectivas teóricas en torno de las formas de definición de las unidades de producción predominantes en el territorio provincial. Además, se describen las principales transformaciones que se producen en la estructura agraria provincial durante los últimos 50 años y se presenta el fenómeno de la complejización de la trama socio-productiva a través de la emergencia de nuevos actores económicos en el agro desde la década de 1990. Finalmente, el artículo se cierra con algunas reflexiones en torno del proceso analizado.

\section{PARTE I}


La Provincia de La Pampa ocupa una superficie de $143.440 \mathrm{~km} 2$ en el centro geográfico de la Argentina (Figura 1). Su ubicación en la zona de transición entre la región central, la pampeana, cuyo, y la Patagonia explica el hecho de que puedan hallarse en ella características propias de cada una de esas regiones. Sin embargo, desde el punto de vista político, la provincia tiene mayor inserción en la región patagónica.

La gradual disminución de las precipitaciones en sentido noreste-sudoeste ha sido un factor determinante en la definición de la organización territorial, la distribución de la población y la actividad económica de la provincia (Figura 2). Así, mientras casi dos tercios de la superficie provincial se ubican en la región árida y semiárida, casi el 80\% de la población se concentra en la porción oriental subhúmeda.

\section{Fase I: La "Conquista del desierto" (1879-1882)}

Las campañas al "desierto" (1879-1882), al tiempo que permitieron la expansión de la antigua frontera virreinal y la consolidación del novel estado argentino, a partir del exterminio de "la amenaza del indio", garantizaron, también, la incorporación de tierras aptas para dar respuesta a la creciente demanda externa de productos agropecuarios. La tendencia al alza en los niveles de demanda internacional de productos agropecuarios -en especial el cuero y la carne salada- constituyó un significativo estímulo para la apropiación extensiva del territorio a través de la consolidación de la estancia ${ }^{2}$ como sistema productivo predominante (Figura 3).

En 1885 los participantes de la "Conquista del Desierto" recibieron bonos de propiedad que fueron rápidamente vendidos a los antiguos terratenientes, profundizándose de esta manera la tendencia a la concentración de la tierra. Esta estructura de propiedad latifundista, que tiende a considerar el suelo como bien de especulación más que como factor de producción, fue clave en la configuración territorial pampeana, que, en este sentido, no se diferencia de lo acontecido en el proceso de ocupación del territorio argentino en general. Luego de la "Conquista", y como resultado de la valorización de la tierra, los propietarios terratenientes comenzaron a desempeñar un papel relevante como agentes económicos (Figura 4).

\section{Fase II: Oleadas migratorias (1882-1945)}

El territorio comprendido por la actual Provincia de La Pampa recibió dos grandes oleadas migratorias, asociadas a dos grandes avanzadas: la militar a través de la "Conquista del Desierto", que fue garantía para la avanzada del capital, montada en un tren de carga.

El primer núcleo poblacional de la provincia se constituyó apenas finalizada la "Conquista del Desierto": Victorica, fundada en 1882 en torno de uno de los "fortines de avanzada". En 1884 se declara oficialmente la creación del Territorio Nacional de La Pampa. Desde entonces y hasta finalizar el siglo XIX se fundaron nuevos núcleos

\footnotetext{
${ }^{1}$ Según estimaciones se calcula entre 10.000 y 12.000 personas la población indígena establecida en el actual territorio de la Provincia de La Pampa antes de 1879.

${ }^{2}$ Grandes establecimientos dedicados a la cría extensiva de hacienda vacuna o lanar.
} 
poblacionales en torno de casas de comercio, boliches de campaña, postas de mensajerías, esquineros de lotes, estancias, etc. (Figura 5). Por entonces la agricultura se limitaba a una mínima producción destinada a la subsistencia. La actividad pastoril, considerada la única posibilidad de valorización de los recursos existentes, se convirtió rápidamente en ganadería comercial, primero ovina y luego vacuna.

Con el comienzo del siglo XX y la llegada del ferrocarril se inicia la segunda oleada de población (Figura 6). El periodo que va de 1900 a 1914 es considerado por algunos como "la época de oro de la colonización agraria pampeana", en solo 19 años ${ }^{3}$ se cuadruplicó la población; inmigrantes de las mas diversas nacionalidades -entre las que destacan los "alemanes del Volga"- fundaron colonias agrícolas en torno de las estaciones, en las tierras del este provincial. Así, la expansión del ferrocarril estructuró la configuración geográficamente dispersa, con pueblos cada 15 o $20 \mathrm{~km}$., que se ha mantenido hasta la actualidad.

En el oeste, trabajadores provenientes de diversas provincias respondieron a la demanda de mano de obra. La actividad predominante era la cría de ganado lanar. A la zona del caldenal (centro de la provincia) llegaron hacheros, solos o con sus familias, provenientes de otras provincias, como Santiago del Estero o San Luis. El desmonte resultante de la explotación del caldén permitió la incorporación de nuevas tierras para la agricultura. La producción forestal era transportada por la misma línea férrea que, al llegar hasta esta zona, contribuía a la formación de pueblos obrajeros con un gran dinamismo demográfico y económico. Cabe destacar que también llegaron trabajadores "golondrina": changarines, poceros, bolseros, estibadores, puesteros y, entre otros, los encargados de extender el tendido de las líneas férreas. Así, las características diferentes de las actividades agrícola y forestal definieron dos tipos de poblamiento contrastantes: el de los agricultores extranjeros afincados y el de los hacheros criollos nómadas.

La Primera Guerra Mundial pondrá fin a ese período. Luego de 15 años, ante los primeros atisbos de recuperación del crecimiento demográfico y económico, sobrevino la crisis del '30, la lluvia de cenizas de 1932 y un período de fuertes sequías entre 1935 y 1937. Como consecuencia miles de productores se empobrecieron, se endeudaron con bancos, perdieron sus tierras y debieron emigrar. La tendencia demográfica decreciente a escala provincial recién lograría revertirse en 1975.

\section{Fase III: Modernización y desarrollo (1945-1975)}

La operatoria populista de la década peronista (1946-1955) se caracterizó por el renovado impulso a la modernización tecnológica, la inversión en infraestructura de transporte, la redistribución del ingreso agrícola y el ajuste de las reglas institucionales de funcionamiento interno (García, 1973, p. 64). Sin embargo, aún cuando ese proceso de modernización transformó las relaciones sociales de producción, la distribución del ingreso, la tecnología, las redes de comercialización, etc.; no se había propuesto mejorar "las condiciones de funcionamiento de la estructura". De tal modo se mantuvieron intactos: "diversas formas y escalas de marginalidad campesina; la hegemonía de los estratos latifundistas sobre los mecanismos nacionales e internacionales de relación e intercambio; el control exclusivista sobre los recursos institucionales de tecnología y de

\footnotetext{
${ }^{3}$ El periodo intercensal 1895-1914
} 
financiamiento agrícolas; la resistencia política al poder integrador y redistribuidor del Estado" (García, 1973, p. 79-80).

En el caso estudiado, la provincialización (20/07/1951) generó una importante estructura de empleo público, independiente de la actividad agropecuaria, que impulsó la creación de una nueva infraestructura de servicios urbanos en la capital y otras ciudades de importancia regional (Figura 7). Por su parte, la modernización tecnológica hizo disminuir la demanda de mano de obra, profundizando el fenómeno de despoblamiento de las zonas agrícolas, con la consecuente incorporación de gran cantidad de población rural a las formas de vida urbanas. De esta forma se consolidará un sistema urbano concentrado en el Este, con núcleos poblacionales periféricos y enclaves productivos dependientes de la dinámica económica de provincias limítrofes.

A esta etapa corresponde también la Ley $\mathrm{N}^{\circ} 468$, la cual establece pautas para la determinación de unidades económicas acordes con las potencialidades agroecológicas de cada región. (Figura 8) Se entiende por unidad económica "El predio que por su superficie, calidad de la tierra, ubicación, mejoras y demás condiciones de producción, racionalmente trabajado por una familia agraria tipo, que aporte la mayor parte del trabajo y desarrolle las actividades corrientes de la zona, le permita alcanzar un nivel de vida digno y evolucionar favorablemente" (Ley $\mathrm{N}^{\circ} 468 / 75$ ).

\section{Fase IV: Globalización neoliberal (1975-2011)}

El proceso de globalización capitalista iniciado a mediados de los '70 marcó "una nueva etapa en el desarrollo del capitalismo agrario". (Muzlera Klappenbach, s/d, p.1) Esta etapa se caracteriza por el afianzamiento de la mecanización, el mejoramiento genético, el desarrollo de nuevas prácticas agronómicas, la difusión de los agroquímicos, la expansión e intensificación agrícola, la complejización de la trama socio-productiva a partir de la emergencia de nuevos actores económicos y la continuidad del proceso de concentración de tierras (Cabo, 2011, p.17).

La profundización de las políticas neoliberales en la década del '90, etapa signada por la desregulación económica y la retracción del Estado, aceleró los cambios en la estructura socioeconómica agropecuaria, exacerbando el proceso de polarización social. En esta etapa comienza a evidenciarse el impacto de la creciente movilidad del capital a través de la "revaloración desigual de los sistemas y espacios agrícolas subnacionales en función de ventajas comparativas redefinidas en el marco de una mayor apertura al exterior" (Llambí, 1993, p. 261). Mientras la desregulación económica impactó espacialmente a través del incremento de los umbrales mínimos en la escala de producción para mejorar las condiciones de competitividad (1988-2002), la retracción del Estado facilitó el endeudamiento de muchos productores, dado que "el fin de los créditos "blandos", otorgados por la banca pública a los sectores más frágiles" los obligó a tomar los créditos ofrecidos por el mercado de capitales (bancos, cooperativas, fondos de inversión, pools de siembra, etc.)" (Muzlera Klappenbach, s/d, p. 3). Como consecuencia se produjo la desaparición del $10 \%$ de las explotaciones en el periodo 1988-2002, lo cual, al sumarse a la caída del 15,4\% entre 1960 y 1988 , totaliza una disminución del $25 \%$ en poco más de 40 años. 


\section{PARTE II}

En un contexto en el que la voracidad geográfica del capital requiere de una constante expansión y reorganización espacial para garantizar su reproducción ampliada, se torna cada vez más acuciante la situación de las unidades de producción campesinas que pretenden enfrentarse a las nuevas lógicas de desapropiación local y acumulación global. En este sentido, considerando que este tipo de unidades constituyen un rasgo distintivo del oeste pampeano -rasgo que se difumina en el $40 \%$ de la superficie provincial- parece pertinente analizar desde diversos ángulos las características distintivas de las UPCs e indagar respecto del fenómeno de su persistencia, contrastándolas con otros tipos de unidades de producción familiar.

\section{Perspectivas teóricas en torno de las unidades de producción campesinas (UPCs)}

La perspectiva marxista clásica entiende las UPCs como un fenómeno residual de la época feudal, el propio Marx "concebía al campesinado como un fenómeno transitorio en el capitalismo, el vestigio de un pasado pre-capitalista" (Llambí, 1990, p. 33). Desde la economía política, en consonancia con la visión chayanoviana, se concibe al campesino como un agente económico orientado esencialmente a "garantizar la subsistencia." El enfoque neomarxista, por su parte, explica el fenómeno de la persistencia campesina por su carácter "funcional" al capitalismo y propone incorporar al campesinado, como categoría económica, dentro del conjunto más amplio de "pequeños productores mercantiles" orientados hacia la maximización de ingresos (Murmis, 1980). Quienes ven al campesino como un productor no muy distinto de otros que operan más claramente bajo el sistema capitalista, buscando maximizar sus ingresos, entienden que su característica diferencial se basa en una "racionalidad campesina", según la cual, el criterio mínimo de eficiencia estaría dado por el logro de la reproducción simple. La reinversión del excedente en medios de producción adicionales significaría la reproducción ampliada del capital, lo cual, en el largo plazo, implica la ruptura del modelo inicial de Unidad de Producción Campesina. La sostenibilidad del proceso de capitalización conlleva la transformación de la unidad campesina en empresa capitalista (Llambí, 1990).

Para Murmis, la unidad de producción campesina se caracteriza por una particular combinación de tierra y trabajo familiar en el proceso productivo, ambos recursos fundamentales, y por una utilización exclusiva de la fuerza de trabajo familiar cumplimentando actividades agropecuarias y no agropecuarias- al interior de la unidad económica familiar. El autor identifica también "unidades de capitalismo campesino", en ellas se halla presente "la relación constitutiva de la explotación capitalista: contratación de asalariados, unido a control de medios de producción y/o a uso de capital para el acceso a la tierra misma" (Murmis, 1980, p.12).

Según Llambí, el criterio fundamental para la definición de unidades de producción es la relación de trabajo que se establece al interior del proceso productivo. El tipo ideal de unidad de producción campesina es, para el autor, aquella que se encuentra en posesión de los medios de producción, cuenta con relativa autonomía para establecer relaciones con otros agentes económicos, definir las características de la producción y adquirir los insumos imprescindibles en el proceso productivo, y además, es autosuficiente en cuanto a la demanda de fuerza de trabajo. A partir de ese tipo ideal define las "Unidades 
de Producción Campesinas insertas en el Sistema Capitalista" como aquellas unidades fundamentalmente agrícolas y mercantiles, en las que imperan relaciones de trabajo basadas en el aporte de fuerza de trabajo de los miembros de un grupo doméstico o "familiar", sin excluir la posibilidad de la contratación -temporaria o permanente- de un número reducido de personas, siempre y cuando tales "relaciones de producción salariales" no constituyan un mecanismo de valorización del capital.

El tipo ideal de UPC se torna endeble ante la presión constante por la subordinación de la unidad respecto de agentes económicos externos, así como también por el desarrollo de una progresiva pérdida de control sobre el proceso productivo y la gestión de la

unidad (subsunción). Para establecer una distinción conceptual entre subsunción y subordinación, Llambí aclara: "mientras que la "subsunción" ocurre a nivel de la esfera de producción, el concepto de subordinación lleva implícito una auténtica relación en la esfera de la circulación de mercancías (tierra, productos, fuerza de trabajo, dinero)." (Llambí, 1980, p.143) En este sentido, en las 3 grandes regiones en que se divide el territorio pampeano (este, centro y oeste) pueden observarse diferentes situaciones de subsunción y subordinación al sistema.

\section{Empresas agropecuarias familiares: unidades tipo "farmer" y UPCs}

Las UPC, características del oeste, y las de tipo farmer, propias del este, constituyen ambas una categoría que podríamos denominar, siguiendo a Caballero, como "empresa familiar", es decir, por oposición a la "empresa capitalista", aquel tipo de explotación en la que el trabajo familiar predomina por sobre el asalariado. Sin embargo, a los fines de profundizar la caracterización, acompañamos al autor en su propuesta de distinguirlas en función de sus diferencias en cuanto a:

a) El grado de capitalización: "Una empresa altamente capitalizada -tipo farmer- tiene, por fuerza, una relación muy distinta con la naturaleza y con la sociedad que otra poco capitalizada -tipo campesina. (...) La segunda razón para distinguir la empresa campesina de la farmer es que, aunque se trate en ambos casos de empresas familiares, las familias en cuestión son muy distintas, y es distinta también la relación entre familia y empresa" (Caballero, 1984, p. 6-7).

b) La forma como se realiza el cálculo económico de la empresa: "Lo que caracteriza el cálculo económico de la empresa campesina (...) es la ausencia de partición del ingreso neto o valor agregado de la empresa en los componentes capitalistas "salario" y "ganancia". Coinciden aquí Marx y Chayanov. En el cálculo económico del campesino -a diferencia del del capitalista- los recursos no aparecen como un capital que, en cuanto tal, puede aspirar a obtener una retribución, una ganancia. Para el campesino, sus recursos aparecen como un campo de aplicación de su propio trabajo" (Caballero, 1984, p. 9).

La diferenciación entre unidades de tipo farmer y UPCs puede establecerse también en función de la autopercepción de los actores sociales referidos respecto de la posición de inclusión o exclusión que ocupan en el sistema capitalista moderno. En palabras de Murmis: "(...) hay en la conceptualización que diferencia campesinos y empresarios no sólo la dimensión que tiene que ver con la incorporación de tecnología o capital y el predominio del cálculo de rentabilidad sino también una dimensión que tiene que ver 
con la visión de exclusión-inclusión en un sistema social moderno. Esto lleva a cierta imagen en la cual los incluidos están de alguna forma instalados en la modernidad capitalista y han llegado para quedarse. Esta imagen se refuerza con la visión según la cual la inclusión ha sido reforzada por políticas estatales que favorecen la incorporación de capital y a las unidades que incorporan capital" (Murmis, 1994, p. 34). Esta perspectiva puede complementarse con la concepción de la "incomunicación rural", entendida como el fenómeno por medio del cual a los campesinos se les impide participar en la "generación del poder político", son excluidos de los "procesos de transmisión de tecnologías y tipos racionales de cultura" y de los mercados (García, 1973, p. 61). En este sentido, Llambí expresa: "Las fuerzas del mercado, los factores de poder, y el peso de las tradiciones culturales obligan a la mayor parte de los pequeños productores a producir con tecnologías tradicionales los productos de consumo directo y baja remuneración que los empresarios agrícolas y complejos agroindustriales no están interesados" (Llambí, 1990, p. 37).

\section{Reconfiguración de la estructura socioterritorial agraria en La Pampa}

La estructura social agraria tradicional de La Pampa, compuesta principalmente por grandes estancieros, colonos o chacareros, "puesteros" y diversos agentes comerciales (proveedores, acopiadores, transportistas, etc.) se complejizó con el advenimiento de nuevos actores económicos en las actividades agropecuarias. La emergencia de los "pools de siembra", en el último lustro de la década del '90, al elevar los precios de la tierra, provocó la desaparición de muchos productores arrendatarios o los obligó a transformarse en contratistas rurales ${ }^{5}$. Los productores propietarios buscaron expandir la escala de producción, adoptando actitudes empresariales, o bien se replegaron y cedieron parte de la tierra a los pools. En este sentido, mientras el CNA 1988 mostraba que, independientemente del tamaño de la explotación, aproximadamente el 80\% de las EAPs eran explotadas por sus propietarios (Cuadro 1); en el último decenio un número importante de pequeños productores propietarios no han podido permanecer al frente de sus unidades y han debido vender sus tierras o, en el mejor de los casos, ceder la explotación de las mismas a sectores capitalizados del agro, convirtiéndose paulatinamente en rentistas con residencia urbana (Aparicio et al., 1992; Slutzky, 2006).

La concentración y la deslocalización de la propiedad de la tierra durante los 90' puede explicarse, aludiendo a dos procesos simultáneos; la compra de tierras por parte de actores sociales ajenos al sector -profesionales residentes en grandes urbes- a los

\footnotetext{
${ }^{4}$ Waldo Ansaldi (1991) define al chacarero como una clase de productor rural, que utiliza su fuerza de trabajo y la de su familia (y puede utilizar -o no- fuerza de trabajo asalariada), con tecnología propia o alquilada a un contratista, que es arrendatario y/o mediero y que, finalmente puede, al acumular capital, transferir una parte de sus ingresos al terrateniente en forma de renta. Se diferencia del farmer por la propiedad de la tierra y de los campesinos por su acumulación de capital, el uso de mayor y mejor tecnología y de mano de obra asalariada. Su enfoque privilegia una clara demarcación de los actores en el terreno, definiéndose la clase de chacarero en oposición a la clase que sí tiene la tierra y que usufructúa con su renta, el terrateniente, oposición sobre todo tangible en la región pampeana.

${ }^{5}$ La figura del contratista toma relevancia como vehiculizador de la "mecanización total de las tareas de cosecha" y la "generalización de la aplicación de agroquímicos y fertilizantes" (Lodola, 2008, p.39). En este sentido, cabe aclarar que, en su carácter empresarial como prestador de servicios agrícolas, el contratista actual en nada se parece al contratista rural familiar de antaño. Asimismo, el tipo de contratista referido aquí tampoco se ajusta estrictamente, en cuanto al nivel de capital acumulado y a sus oportunidades para realizarlo, al descripto por Aparicio (1992) como "nuevo empresario agrario".
} 
productores endeudados o en quiebra; y la adquisición de tierras, en general mediante alquiler, por parte de grandes grupos económicos nacionales o extranjeros con el objetivo de trabajarlas a gran escala (Sili, 2005; Lodola, 2008). El ingeniero Sergio Cabo, analizando la evolución del proceso en el este pampeano, plantea: "En las ultimas campañas agrícolas se observa la aparición de inversores de riesgo que acceden a la tierra por medio del arrendamiento, el cual puede ser pactado por un ciclo agrícola o por un periodo más prolongado (tres a cuatro años), pero en cualquier caso coincide en pactar el pago de valores elevados por hectárea para sembrar cultivos de soja o girasol" (Cabo, 2011, p. 18). Esta competencia por la tierra, cuya manifestación en el mercado se expresa a través de la elevación del valor de los alquileres, se resuelve siempre en desmedro de los pequeños arrendatarios.

Los datos estadísticos acumulados desde la etapa de la "modernización tecnológica" dan cuenta de la reconfiguración de la estructura agraria pampeana. Este periodo se caracteriza por una fuerte tendencia a la concentración de la propiedad de la tierra, con disminución del número de productores por la desaparición de una gran cantidad de explotaciones ubicadas en los estratos de menores superficies. La disminución en el total de explotaciones para el periodo 1960-1988 se explica enteramente por la reducción del estrato de EAPs $^{6}$ con superficies menores a las 200 has. En el mismo periodo se incrementa la cantidad de EAPs ubicadas en todos los estratos superiores a ese umbral (Cuadro 2). Por su parte, entre 1988 y 2002 se observa una fuerte disminución en el número de EAPs con superficies menores a las 500 has., así como la retracción -porcentualmente menos significativa- de las EAPs con superficies entre 5.000 y 10.000 has. Solo muestran un incremento digno de mención las EAPs que se ubican en el estrato que va de las 1.000 a las 5.000 has. (Cuadro 3). La escala de extensión, en 2002, también daba cuenta de la concentración: el 7\% de las EAPs se repartían el $48 \%$ de la superficie ocupada, mientras que el $51 \%$ de las EAPs sumaba apenas el 6,6\% de la superficie ocupada (Cuadro 4). Entre las provincias que componen la región pampeana, La Pampa es la única que sufrió un incremento en el número de explotaciones agropecuarias consideradas pobres en el periodo 1988-2002 (Cuadro 5). Por otra parte, las sociedades (SRL, SA, SCA) son la forma jurídica de propiedad de la tierra que más se ha incrementado $(23,4 \%)$ en el periodo 1988-2002, proceso denominado como "empresarización" (Cabo, 2011) (Cuadro 6). En este sentido, resulta relevante considerar a Murmis cuando sostiene: "El elemento fundamental para caracterizar al sector empresario es precisamente su capacidad de invertir capital de acuerdo con el proceso de modernización ligado a un complejo circuito que lo rodea "por atrás" y por "adelante" (Murmis, 1994, p. 26).

El análisis de la composición de la estructura agraria es una muestra de que los diferentes grupos sociales no se encuentran en iguales condiciones para apropiarse del excedente económico generado a partir del progreso tecnológico (Piñero, 1975, p. 3). Esto ocurre porque "la capacidad de adopción no es el resultado de los deseos y motivaciones individuales de los productores, sino que lo es de la capacidad real que tienen para hacerlo, lo cual es consecuencia de la situación diferencial en cuanto a

\footnotetext{
${ }^{6}$ Unidad de organización de la producción, con una superficie no menor a $500 \mathrm{~m} 2$, dentro de los límites de una misma provincia, que independientemente del número de parcelas (terrenos no continuos) que la integren: produce bienes agrícolas, pecuarios o forestales destinados al mercado, tiene una dirección que asume la gestión y riesgos de la actividad y utiliza en todas las parcelas que la integran, los mismos medios de producción de uso durable y parte de la misma mano de obra.
} 
dotación de recursos, capacidad financiera, nivel cultural inicial, etc., que cada uno tiene y que son restricciones objetivas que ningún productor puede resolver por si mismo" (Piñero, 1975, p. 15).

En todo el territorio provincial se están produciendo vertiginosas transformaciones en una espacialidad social que se ha venido construyendo durante más de un siglo. En el este, esas transformaciones "se manifiestan en un gradual aumento de la heterogeneidad de la estructura productiva y además, en una pérdida de capacidad de acción de los pequeños y medianos productores rurales para organizar adecuadamente el ámbito de gestión de sus propias explotaciones rurales"(Shmite, 2003, p. 38). En el caso de las zonas netamente ganaderas (de cría) de la provincia, las situaciones críticas han reflejado el fenómeno de regresión tecnológica defensiva que describe Murmis para la pampa húmeda: "Explotaciones ganaderas, sobre todo medianas, que habían incorporado tecnología, como uso de pasturas y alambrados eléctricos, alquilaron sus campos o la parte ganadera de éstas a empresarios" (Murmis,1998, p. 221). Sin embargo, el relacionamiento asimétrico entre unidades de producción de distinto tipo, así como con otros agentes económicos es más evidente en el oeste. La polarización deja ver allí más claramente que "lo que esta en juego (...) no es tan solo el posible "control" o dominio de un proceso productivo ajeno, sino las posibilidades de: a) extracción de excedentes, b) generación de ganancias mercantiles extraordinarias y c) extracción de parte de la capacidad productiva de la unidad por medio de un flujo o transferencia de su fuerza de trabajo" (Llambí, 1990, p. 127 y 143). A su vez, la posición relativa más ventajosa de los agentes económicos capitalistas (especuladores inmobiliarios, instituciones bancarias, intermediarios comerciales) en cuanto a la dotación de recursos les permite ubicar bajo su órbita a las unidades que resultan desfavorecidas -en nuestro caso las UPCs y los arrendatarios sin tierra propia-.

\section{REFLEXIONES FINALES}

El proceso de incorporación del territorio pampeano a los espacios del capital, así como su actualización, exige ser observado desde una perspectiva geohistórica que permita analizar la articulación trialéctica entre territorialización, desterritorialización y reterritorialización (TDR) de los sistemas de dominación social predominantes. En el caso estudiado, el sistema de dominación social, asociado inicialmente a una estructura agraria latifundista, se configuró a través de la monopolización de la tierra, la subordinación de las fuerzas productivas y el control hegemónico de los mecanismos de representación y organización política. Así, la especulación inmobiliaria impulsó la valorización de la tierra y acentuó el "proceso de concentración latifundista". Luego, la modernización tecnológica profundizó "la brecha entre la agricultura comercial, las empresas latifundistas y el enorme aluvión de pequeñas explotaciones campesinas" (García, 1973, p. 95). Finalmente, en la etapa actual, la profundización de la polarización entre el incremento de las articulaciones agroindustriales y la progresiva pérdida de autonomía por parte de los productores agropecuarios (Teubal y Pastore, 1995), se destaca como uno de los rasgos característicos de la problemática agraria en el capitalismo globalizado. En este sentido, la disputa entre la adaptación y la resistencia, confirma que la reconfiguración en curso no ha trastocado las bases ideológicas y normativas del sistema de dominación social más que para ajustarlo a las nuevas tramas de relaciones sociales. 
En la medida que las contradicciones de la forma de organización social capitalista se profundizan, su materialización a través de la disputa por la apropiación del espacio geográfico se actualiza. La totalidad compleja de ese proceso muestra, por una parte, un rostro inocultable que se torna evidente a partir del estudio de las transformaciones en la estructura agraria y en la composición social de los espacios rurales. Sin embargo, aunque con intensidades variables, el proceso de dominación de las formas no capitalistas de organización social nunca se ha detenido. Subyace, como complemento, una compleja estructura argumentativa -sustentada en "irrefutables confirmaciones de base técnica"- que da sustento a una suerte de profecía autocumplida acerca del avance inexorable del capital. ¿El "destino manifiesto" del capital transnacional?

\section{BIBLIOGRAFÍA}

APARICIO, Susana; GIARRACCA, Norma; TEUBAL, Miguel. Las transformaciones en la agricultura: el impacto sobre los sectores sociales. En SAUTÚ, R; JOUVAT, J. (comps.). Después de Germani. Exploraciones sobre la estructura social agraria, Buenos Aires: Editorial Paidós, 1992.

ANSALDI, Waldo. La pampa es ancha y ajena. La lucha por las libertades capitalistas y la construcción de los chacareros como clase. Unidad de Docencia e Investigación Socio-Históricas de América Latina (UDISHAL), sin datos, 1991.

CABALLERO, José María. Campesinos y farmers: desarrollo capitalista y tipo de empresa agraria. Roma: FAO, 1984.

CABO, Sergio. Transformación territorial del espacio rural del centro-este de La Pampa. La Pampa: Ediciones INTA, 2011.

GARCÍA, Antonio. Sociología de la reforma agraria en América Latina. Buenos Aires: Amorrortu Editores, 1973.

Gobierno de la Provincia de La Pampa. Ministerio de la Producción. Anuario Estadístico. La Pampa, 2010.

Gobierno de la Provincia de La Pampa. Subsecretaría de Planificación. Sili, Marcelo (Dir). Diagnóstico de situación de la provincia de La Pampa. La Pampa: Editora L\&M, 1998.

Instituto Nacional de Estadísticas y Censos. Censo Nacional Agropecuario, 2002

LLAMBÍ, Luis. Las unidades de producción campesina en un intento de teorización. $V$ Congreso Mundial de Sociología Rural. México, D.F., agosto 7-12, 1980.

LLAMBÍ, Luis. El campesinado en la economía política, Estudios Rurales, 1990, vol. 13, n० 3, pp. 209-241.

LLAMBÍ, Luis. Reestructuración mundial y sistemas agroalimentarios. Necesidad de nuevos enfoques. Comercio Exterior, 1993, vol. 43, n 3 . 
LODOLA, Agustín. Contratistas, cambios tecnológicos y organizaciones en el agro pampeano. Santiago de Chile: CEPAL, 2008.

MURMIS, Miguel. Tipología de pequeños productores campesinos en América Latina. San José, Costa Rica: IICA. - Promer, 1980.

MURMIS, Miguel: Algunos temas para la discusión de la Sociología Rural Latinoamericana. Reestructuración, desestructuración y problemas de excluidos e incluidos. Ruralia, 1994, n ${ }^{0}$ 5, p. 43-68.

MURMIS, Miguel: El agro argentino: algunos problemas para su análisis. En GIARRACCA, Norma y CLOQUELL, Silvia. (Comps.). Las Agriculturas del Mercosur. Buenos Aires: Editorial La Colmena - CLACSO, 1998.

MUZLERA KLAPPENBACH, José. Las recomposiciones de la producción agropecuaria familiar a comienzos del siglo XXI. Proyecto de Tesis Maestría IDESUNGS, Buenos Aires, sin datos.

PIÑEIRO, Martín; MARTÍNEZ, Juan Carlos; ARMELÍN, Carlos. Politica tecnológica para el sector agropecuario, INTA, 1975.

SHMITE, Stella Maris. Procesos de construcción de la especialidad social. Un marco teórico-metodológico para el análisis del espacio rural de La Pampa, Argentina. En MEDUS, Norma (Comp.). En torno de lo rural. Matices de la geografía. La Pampa: EDULPam, 2003.

TEUBAL, Miguel; PASTORE, Rodolfo: El agro y los complejos agroindustriales: el caso argentino. En TEUBAL, Miguel; GIARRACCA, Norma y PASTORE, Rodolfo: Globalización y expansión agroindustrial: ¿superación de la pobreza en América Latina? Buenos Aires: Editorial Corregidor, 1995.

SLUTZKY, Daniel. Argentina problemáticas en torno a la tenencia de la tierra. Buenos Aires: Ministerio de Economía y Producción. Secretaría de Agricultura, Ganadería, Pesca y Alimentos, 2006. 
Figura 1. Ubicación relativa de la Provincia de la Pampa, Argentina.

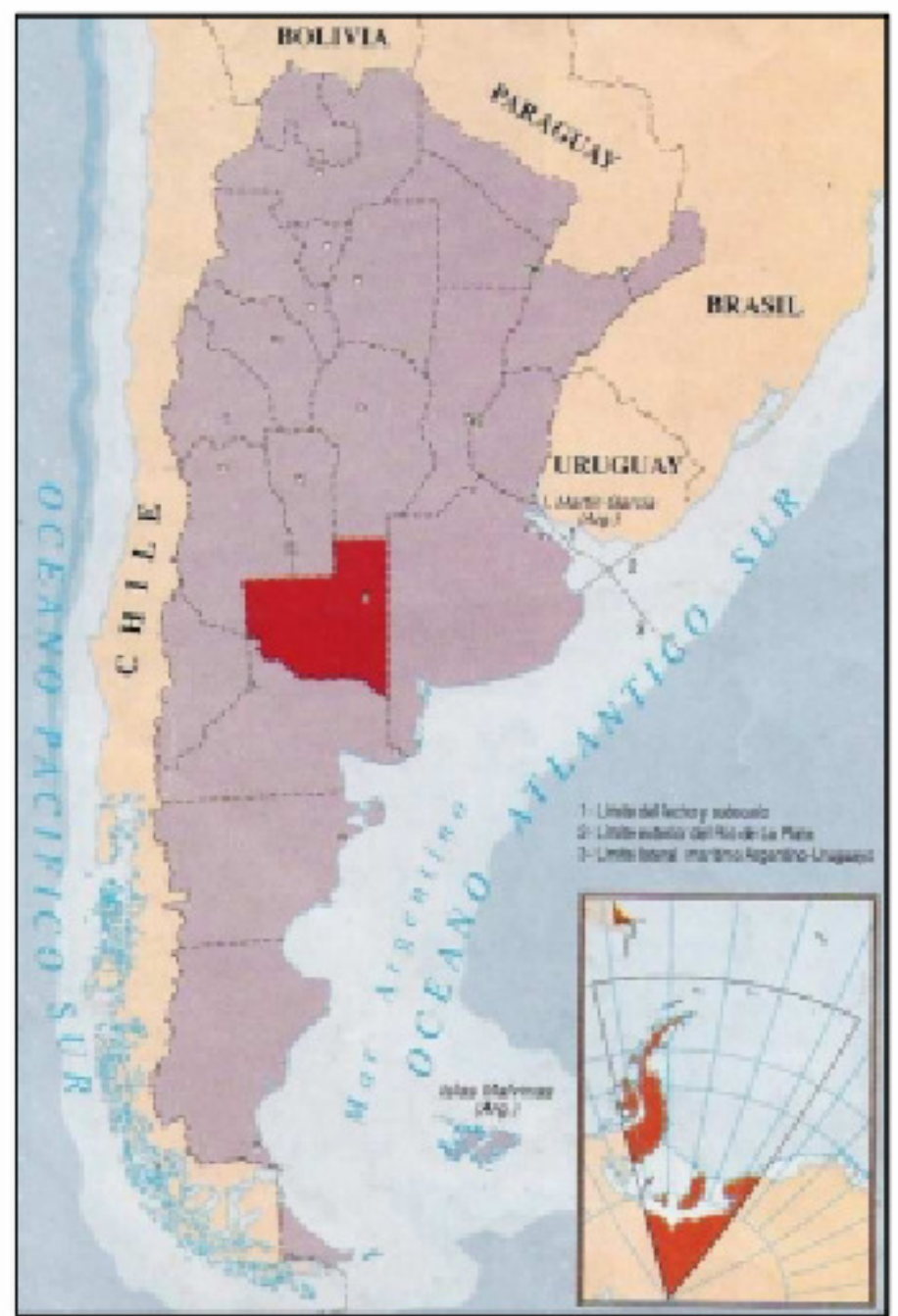

Fuente: $<\underline{\text { http://www.camposdelapampa.com.ar/pdf/Ubicacion-Geografica.pdf }>}$. 
Figura 2. Mapa de isohietas de la Provincia de La Pampa.

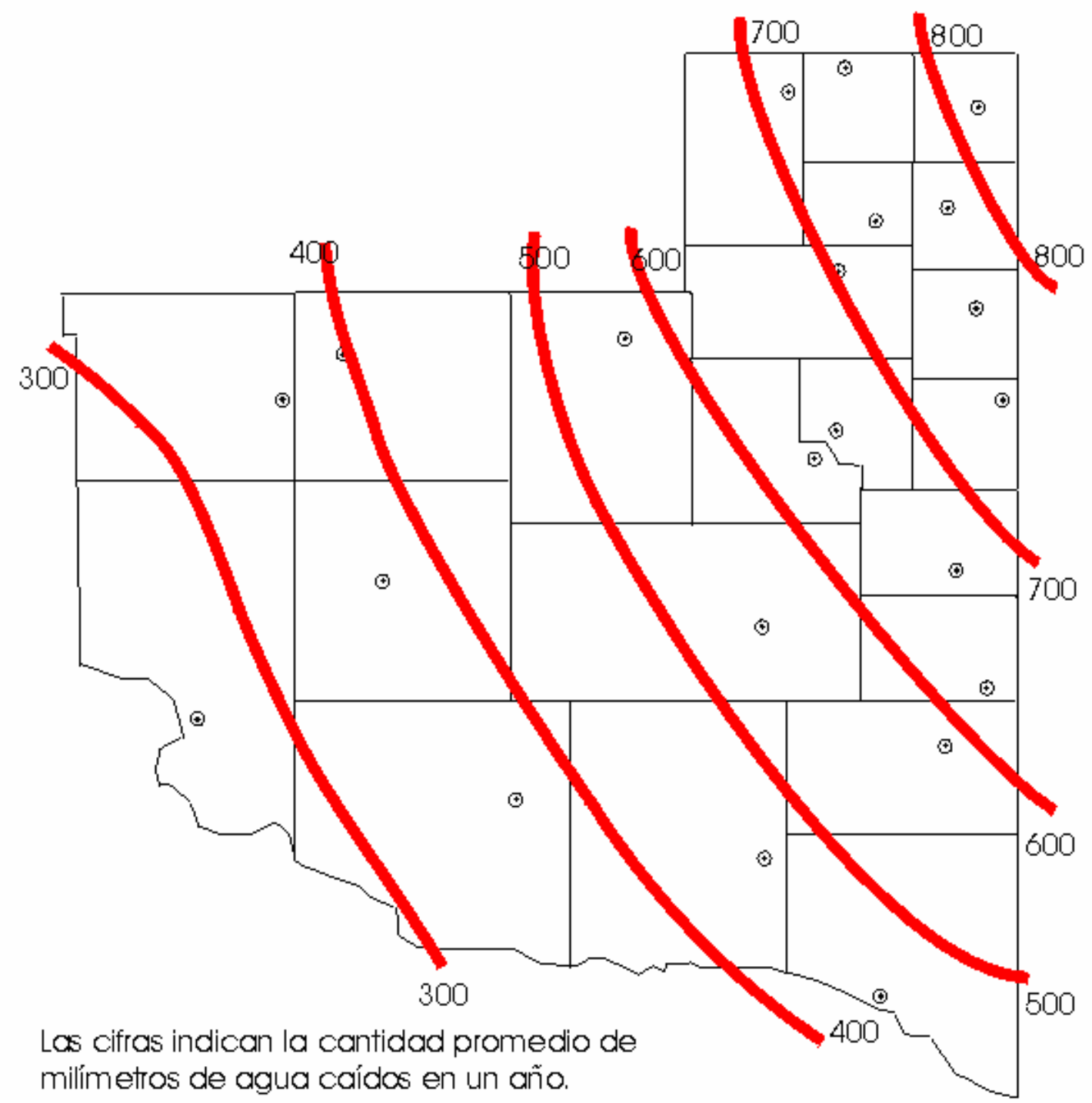

Fuente: Gobierno de la Provincia de La Pampa. Subsecretaría de Planificación, 1998. 
Figura 3. Periodo indígena (hasta 1879).

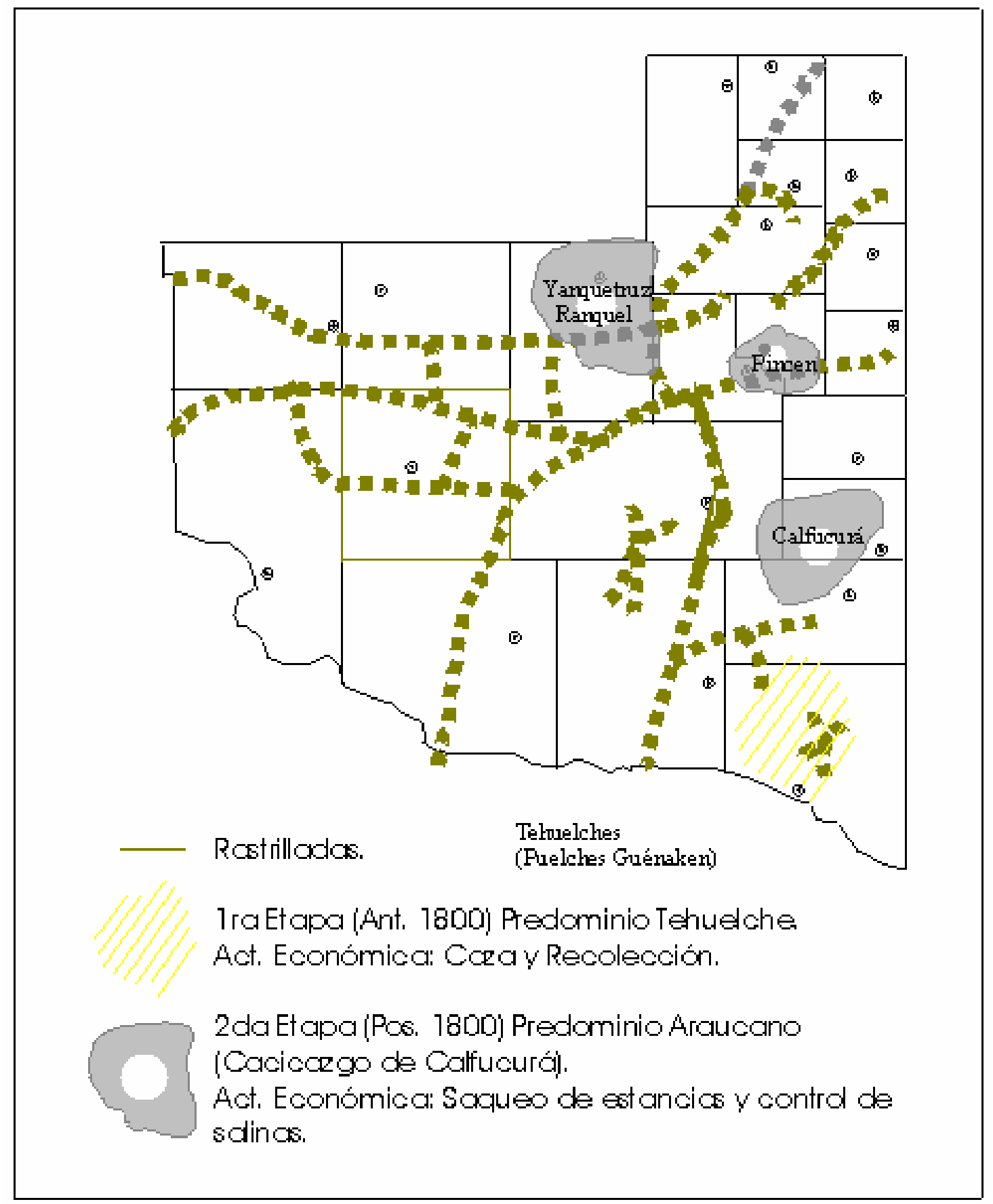

Fuente: Gobierno de la Provincia de La Pampa. Subsecretaría de Planificación, 1998. 
Figura 4. Primera oleada de repoblamiento. 1880-1891.

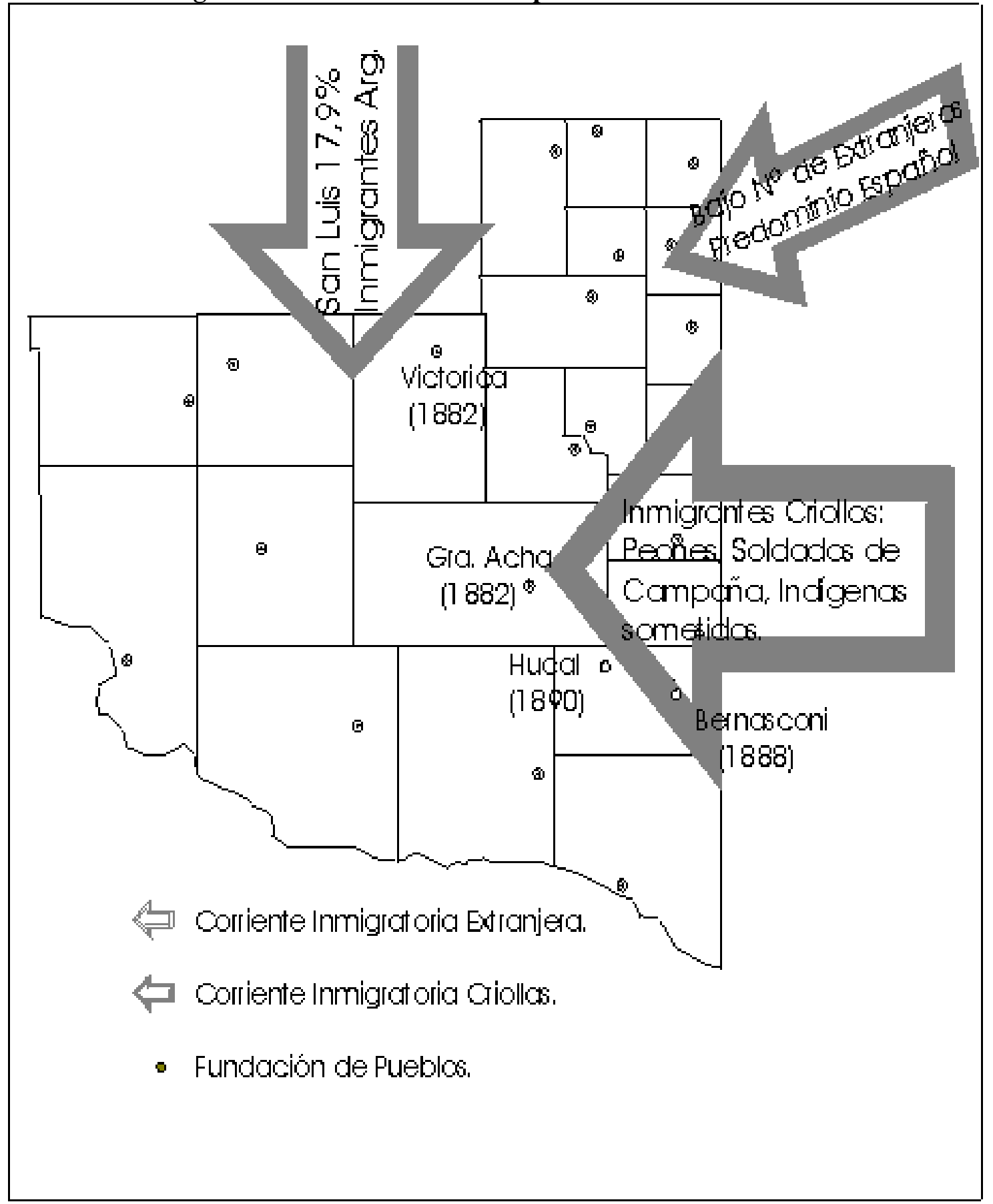

Fuente: Gobierno de la Provincia de La Pampa. Subsecretaría de Planificación, 1998. 
Figura 5. Segunda oleada de repoblamiento. 1892-1899.

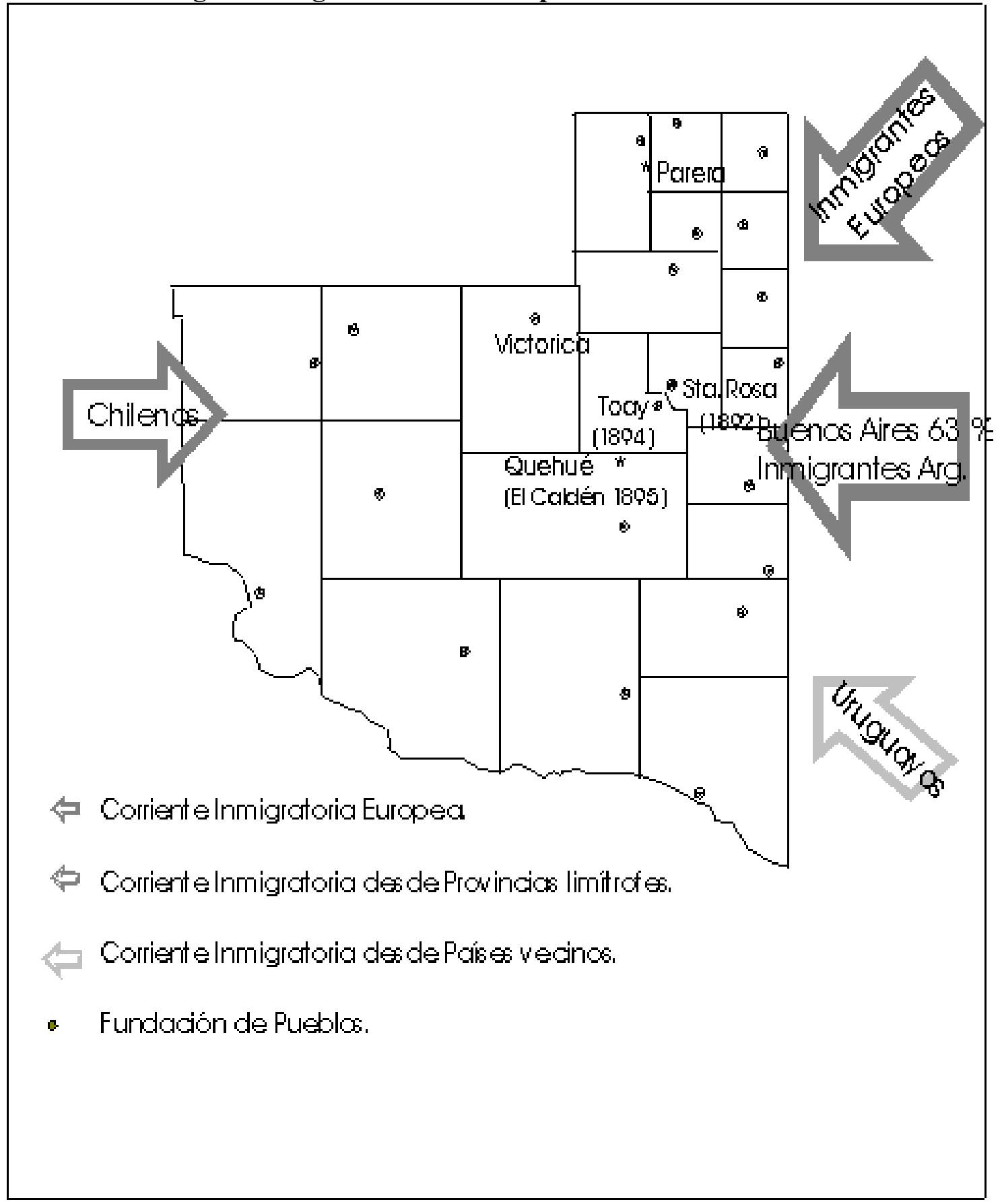

Fuente: Gobierno de la Provincia de La Pampa. Subsecretaría de Planificación, 1998. 
Figura 6. Tercera oleada de repoblamiento. 1900-1914.

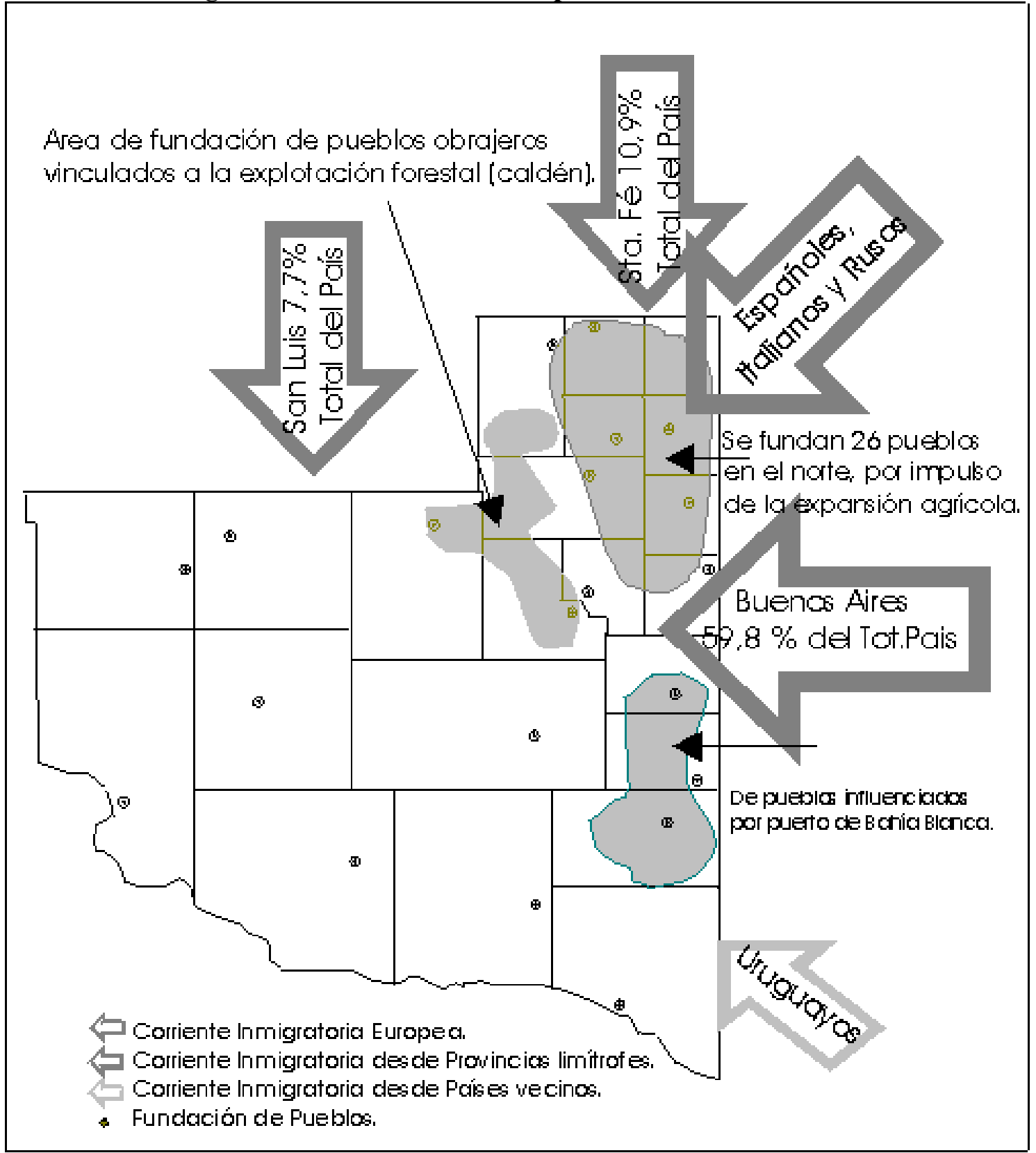

Fuente: Gobierno de la Provincia de La Pampa. Subsecretaría de Planificación, 1998. 
Figura 7. Áreas con crecimiento urbano en el periodo de la provincialización, 1951.

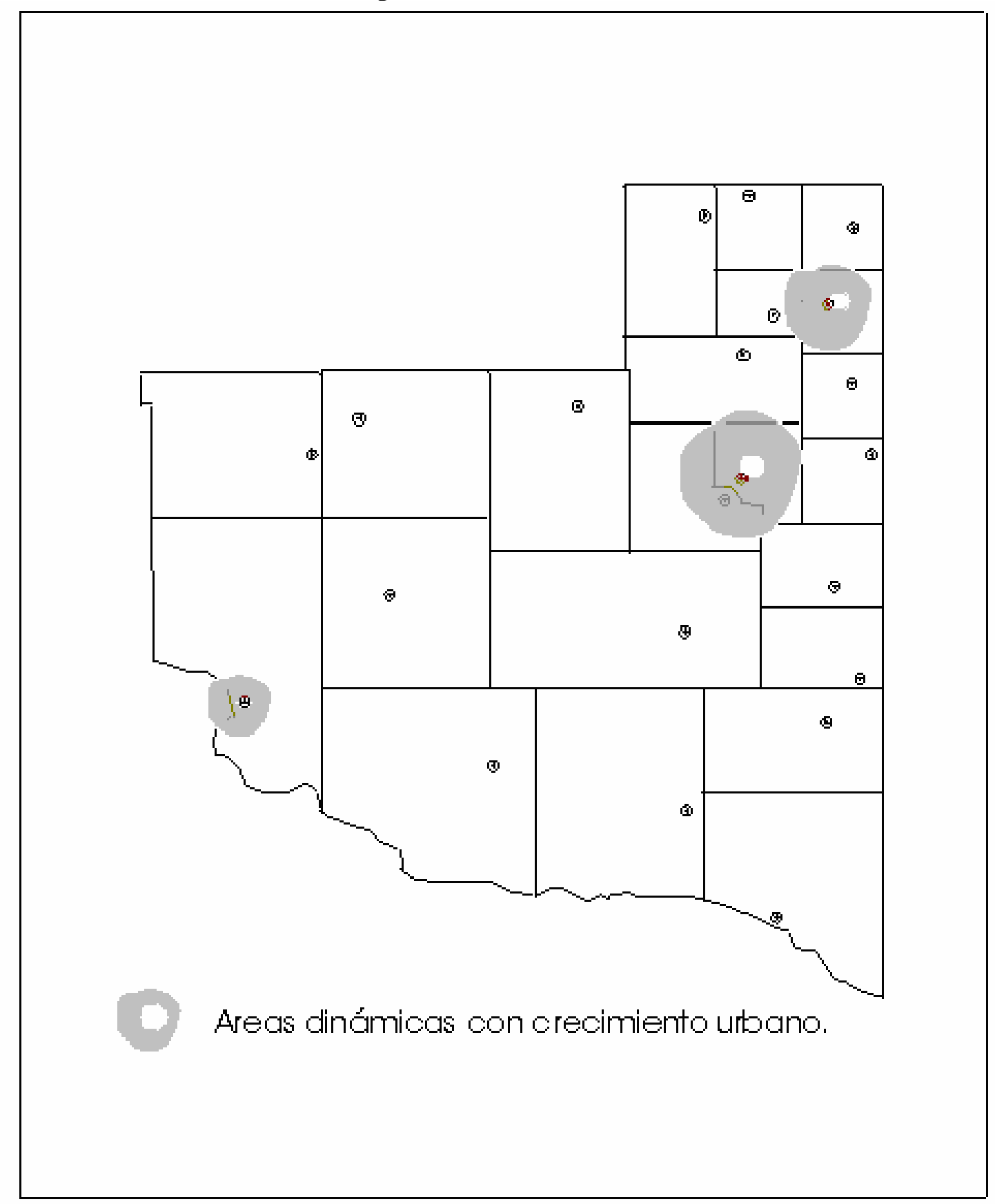

Fuente: Gobierno de la Provincia de La Pampa. Subsecretaría de Planificación, 1998. 
Figura 8. Unidades económicas de la Provincia de La Pampa.

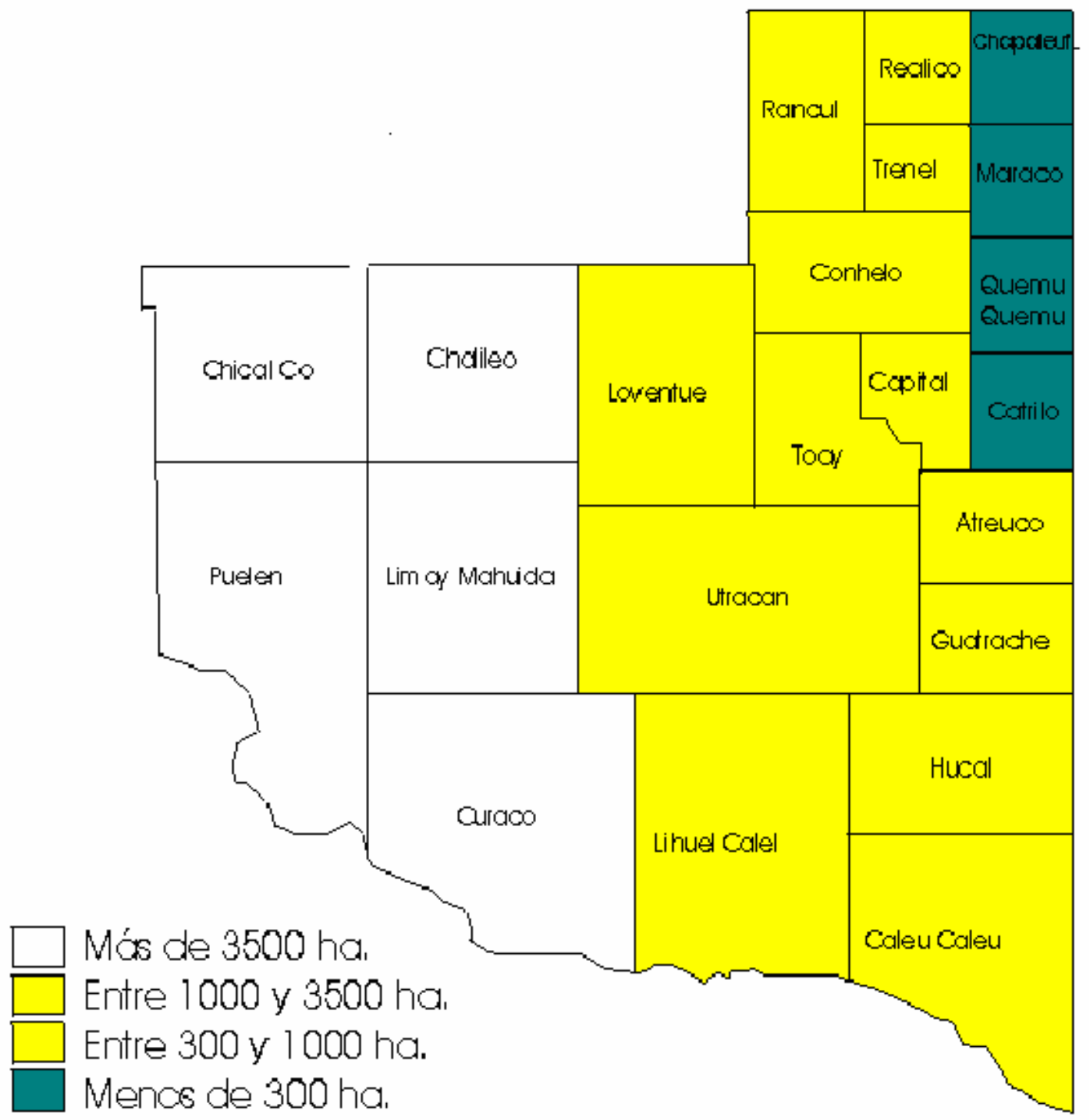

Fuente: Gobierno de la Provincia de La Pampa. Subsecretaría de Planificación, 1998. 
Cuadro 1. Tenencia de la tierra en la Provincia de La Pampa. 1988.

\begin{tabular}{|l|l|l|l|l|}
\hline Régimen de & Hasta & $\mathbf{1 0 0} \mathrm{a}$ & $\mathbf{1 . 0 0 0} \mathrm{a}$ & $\pm \mathbf{1 0 . 0 0 0}$ \\
Tenencia & $\mathbf{1 0 0} \mathbf{~ h a}$ & $\mathbf{1 . 0 0 0} \mathbf{~ h a}$ & $\mathbf{1 0 . 0 0 0}$ ha & ha \\
\hline Propiedad personal & 1.124 & 3.053 & 1.487 & 98 \\
\hline Arrendamiento & 125 & 315 & 116 & 6 \\
\hline Aparcería & 13 & 15 & 5 & -- \\
\hline Contrato accidental & 55 & 108 & 16 & -- \\
\hline Ocupación & 25 & 13 & 190 & 13 \\
\hline Otros & - & 7 & 4 & -- \\
\hline TOTAL & $\mathbf{1 . 3 4 2}$ & $\mathbf{3 . 5 1 1}$ & $\mathbf{1 . 8 1 8}$ & $\mathbf{1 1 7}$ \\
\hline
\end{tabular}

Fuente: Gobierno de La Pampa. Ministerio de la Producción.

Cuadro 2. Estructura agraria en la Provincia de La Pampa. (1960-1988).

\begin{tabular}{|c|c|c|c|c|c|}
\hline & \multicolumn{5}{|c|}{ Estrato de explotaciones (en héctareas) } \\
\hline Bf́tos & menos de 200 & de 200 a 1000 & de 1000 a 2500 & Mas de 2500 & total \\
\hline Afio 1960 & 4258 & 3763 & 1211 & 931 & 10200 \\
\hline Afio 1988 & 1463 & 4717 & 1275 & 1176 & 8631 \\
\hline
\end{tabular}

Fuente: INDEC-CNA, 1988.

Cuadro 3. EAPs en La Pampa y porcentaje de variación intercensal. 1988-2002.

\begin{tabular}{|c|r|r|r||}
\hline & \multicolumn{1}{|l|}{$\begin{array}{l}\text { Total La } \\
\text { Pampa 1988 }\end{array}$} & $\begin{array}{l}\text { Total La } \\
\text { Pampa 2002 }\end{array}$ & $\begin{array}{l}\text { \% de } \\
\text { variación }\end{array}$ \\
\hline $\begin{array}{c}\text { EAP con limites } \\
\text { definidos }\end{array}$ & 8.631 & 7.774 & $-10 \%$ \\
\hline $5,1-10$ & 259 & 113 & $-56 \%$ \\
\hline $10,1-25$ & 238 & 190 & $-20 \%$ \\
$25,1-50$ & 308 & 258 & $-16,23 \%$ \\
\hline $50,1-100$ & 658 & 500 & $-24 \%$ \\
$100,1-200$ & 1.206 & 1.003 & $-16,85 \%$ \\
\hline $200,1-500$ & 2.194 & 1.880 & -14.30 \\
\hline $500,1-1.000$ & 1.317 & 1.320 & $+0,22 \%$ \\
\hline $1.000,1-2.500$ & 1.275 & 1.352 & $+6 \%$ \\
\hline $2.500,1-5.000$ & 631 & 632 & $+0,16 \%$ \\
\hline $5.000,1-10.000$ & 378 & 356 & $-5,8 \%$ \\
\hline$>10.000,1$ & 167 & 170 & $+1,8 \%$ \\
\hline \multicolumn{4}{|c|}{} \\
\hline
\end{tabular}

Fuente: INDEC-CNA, 2002. 
Cuadro 4. Extensión de EAPs, número de productores y superficie ocupada.

\begin{tabular}{|l|c|c|c|c|c|c|c|c|c|c|}
\hline & Rango & $\%$ & Rango & $\%$ & Rango & $\%$ & Rango & $\%$ & Total Rango & Total \% \\
\hline & 5 a 50 has & & $51-500$ has & & 501.5000 & & Mas 5000 & & & \\
\hline EAP (Product) & 561 & 7 & 3.383 & 44 & 3.304 & 43 & 526 & 7 & 7.774 & 100 \\
\hline Sup. Ocupada & 14.023 & 0 & 829.481 & 7 & 5.784 .575 & 45 & 6.106 .929 & 48 & 12.735 .008 & 100 \\
\hline
\end{tabular}

Fuente: INDEC-CNA, 2002

Cuadro 5. EAPs pobres en los Censos de 1988 y 2002, por provincias.

\begin{tabular}{|c|c|c|c|c|c|c|c|}
\hline & \multicolumn{2}{|c|}{ CNA 1988} & \multicolumn{2}{|c|}{ CNA 2002} & \multirow{2}{*}{ 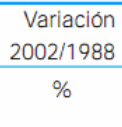 } & 1988 & 2002 \\
\hline & EAP pobres & EAP totales & EAP pobres & EAP totales & & \multicolumn{2}{|c|}{$\begin{array}{l}\% \text { EAP Pobres } \\
\text { /EAP totales }\end{array}$} \\
\hline TOTAL DEL PAIS & 163245 & 421221 & 132672 & 333533 & -19 & 39 & 40 \\
\hline Buenos Aires & 14438 & 75531 & 10596 & 51116 & -27 & 19 & 21 \\
\hline Córdoba & 8958 & 40817 & 5474 & 26226 & .39 & 22 & 21 \\
\hline Santa Fe & 4431 & 37029 & 3610 & 28103 & -19 & 12 & 13 \\
\hline Entre Ríos & 10354 & 27197 & 7806 & 21577 & -25 & 38 & 36 \\
\hline La Pampa & 1245 & 8718 & 1256 & 7775 & 1 & 14 & 16 \\
\hline PAMPEANA & 39426 & 189292 & 28742 & 134797 & -27 & 21 & 21 \\
\hline
\end{tabular}

Fuente: IICA, 2007

Cuadro 6. Formas jurídicas de la propiedad de la tierra, 1988 y 2002.

\begin{tabular}{|c|c|c|c|c|c|}
\hline CNA & Total EAPs & Persona Física & Soc. de Hecho & Sociedades* & Otras formas \\
\hline 1988 & 8.631 & 5.975 & 2.122 & 423 & 111 \\
2002 & 7.775 & 5.674 & 1.530 & 522 & 49 \\
\hline \multicolumn{2}{|r|}{ Var. (\%) } & -5.0 & $-27,9$ & 23,4 & $-55,9$ \\
\hline
\end{tabular}

Fuente: Cabo, 2011, en base a CNA, 1988 y 2002.

* Incluye sociedades anónimas, sociedades en comandita por acciones y sociedades de responsabilidad limitada.

(C) Rodrigo Hernán Alves Rolo, 2012.

(C) GeoGraphos. Revista Digital para Estudiantes de Geografia y Ciencias Sociales, 2012.

\section{Get Graphos}

